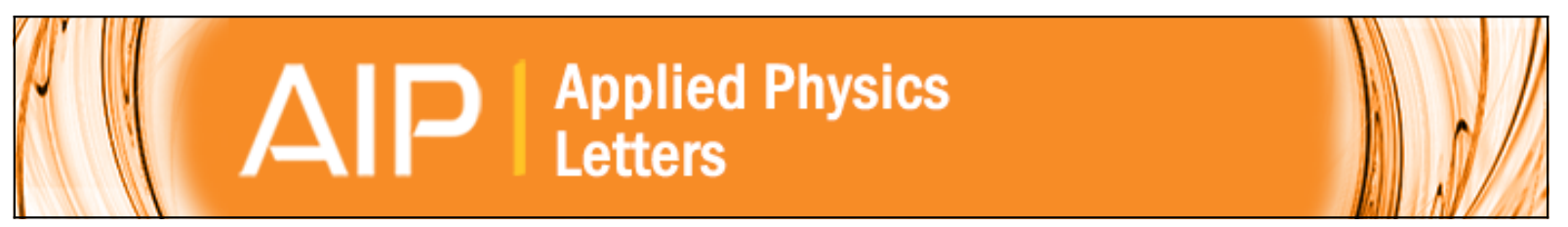

\title{
Holmium hafnate: An emerging electronic device material
}

Shojan P. Pavunny, Yogesh Sharma, Sudheendran Kooriyattil, Sita Dugu, Rajesh K. Katiyar, James F. Scott, and Ram S. Katiyar

Citation: Applied Physics Letters 106, 112902 (2015); doi: 10.1063/1.4915503

View online: http://dx.doi.org/10.1063/1.4915503

View Table of Contents: http://scitation.aip.org/content/aip/journal/apl/106/11?ver=pdfcov

Published by the AIP Publishing

\section{Articles you may be interested in}

Disorder driven structural and dielectric properties of silicon substituted strontium titanate

J. Appl. Phys. 118, 034105 (2015); 10.1063/1.4927042

Band gap enhancement and electrical properties of La 203 films doped with $\mathrm{Y} 2 \mathrm{O} 3$ as high- $\mathrm{k}$ gate insulators Appl. Phys. Lett. 94, 042901 (2009); 10.1063/1.3075954

High-temperature conduction behaviors of $\mathrm{HfO} 2$ / TaN -based metal-insulator-metal capacitors

J. Appl. Phys. 102, 073706 (2007); 10.1063/1.2786712

Characteristics of sputtered Ti $1-x \mathrm{Al} \times \mathrm{N}$ films for storage node electrode barriers

J. Vac. Sci. Technol. B 19, 2289 (2001); 10.1116/1.1421567

Electrical characteristics of metal-dielectric-metal and metal-dielectric-semiconductor structures based on electron beam evaporated Y 203 , Ta 205 and $\mathrm{Al} 2 \mathrm{O} 3$ thin film

J. Appl. Phys. 84, 6747 (1998); 10.1063/1.369002

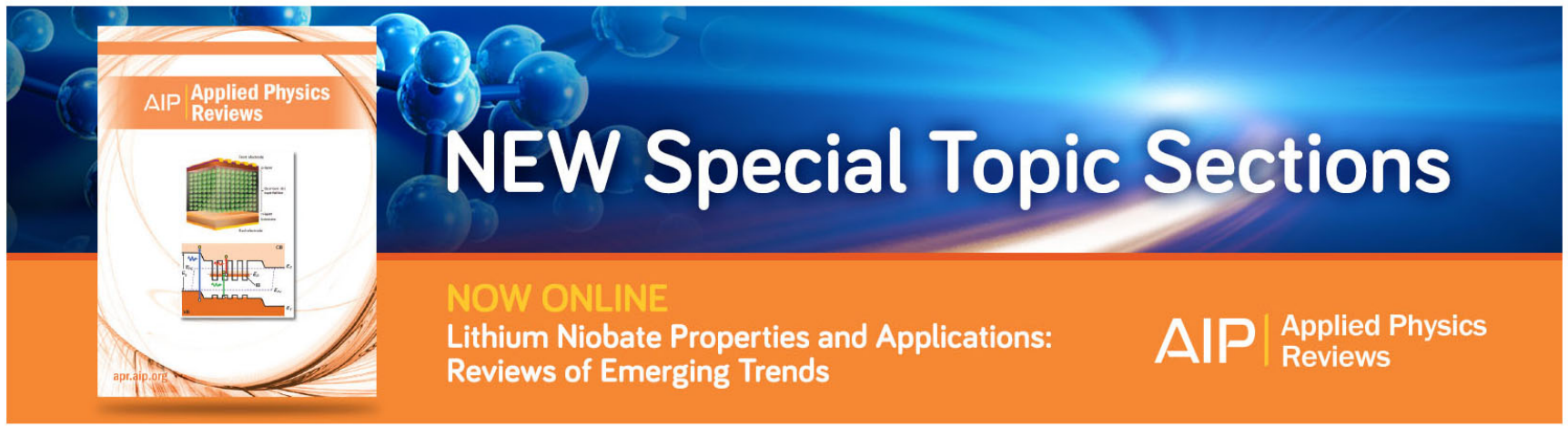




\title{
Holmium hafnate: An emerging electronic device material
}

\author{
Shojan P. Pavunny, ${ }^{1, a)}$ Yogesh Sharma, ${ }^{1}$ Sudheendran Kooriyattil, ${ }^{1}$ Sita Dugu, ${ }^{1}$ \\ Rajesh K. Katiyar, ${ }^{1}$ James F. Scott, ${ }^{1,2}$ and Ram S. Katiyar ${ }^{1, a)}$ \\ ${ }^{1}$ Department of Physics and Institute for Functional Nanomaterials, University of Puerto Rico, \\ P.O. Box 70377, San Juan, Puerto Rico 00936-8377, USA \\ ${ }^{2}$ Department of Physics, Cavendish Laboratory, University of Cambridge, Cambridge CB3 OHE, \\ United Kingdom
}

(Received 20 February 2015; accepted 6 March 2015; published online 17 March 2015)

\begin{abstract}
We report structural, optical, charge transport, and temperature properties as well as the frequency dependence of the dielectric constant of $\mathrm{Ho}_{2} \mathrm{Hf}_{2} \mathrm{O}_{7}(\mathrm{HHO})$ which make this material desirable as an alternative high-k dielectric for future silicon technology devices. A high dielectric constant of $\sim 20$ and very low dielectric loss of $\sim 0.1 \%$ are temperature and voltage independent at $100 \mathrm{kHz}$ near ambient conditions. The $\mathrm{Pt} / \mathrm{HHO} / \mathrm{Pt}$ capacitor exhibits exceptionally low Schottky emissionbased leakage currents. In combination with the large observed bandgap $\mathrm{E}_{\mathrm{g}}$ of $5.6 \mathrm{eV}$, determined by diffuse reflectance spectroscopy, our results reveal fundamental physics and materials science of the HHO metal oxide and its potential application as a high-k dielectric for the next generation of complementary metal-oxide-semiconductor devices. (C) 2015 AIP Publishing LLC.

[http://dx.doi.org/10.1063/1.4915503]
\end{abstract}

Moore's law ${ }^{1}$ which states that the number of transistors per chip doubles approximately every 18 months is the driving force in delivering microprocessors with increased transistor density, faster switching speed, and lower power characteristics from one technology generation to another. ${ }^{2}$ In this regard, downscaling of the metal-insulator-semiconductor (MIS) stacks and metal-insulator-metal (MIM) capacitors is being effected in complementary metal-oxidesemiconductor (CMOS) devices. The most difficult challenge to meet this law is to deliver materials with high density at the nanometer scale. One critical component in high performance logic (e.g., metal-oxide-semiconductor field-effect transistor (MOSFET)) and memory [e.g., dynamic random access memory (DRAM) and resistive RAM (RRAM)] devices is a thin layer of insulator/dielectric oxide material with significantly enhanced physical properties (such as large bandgap, high linear dielectric constant, reduced loss tangent, lower leakage currents, and CMOS process compatibility) in order to continue aggressive scaling. ${ }^{3}$ Under this context, we have developed the ternary oxide material, $\mathrm{Ho}_{2} \mathrm{Hf}_{2} \mathrm{O}_{7}(\mathrm{HHO})$ in order to investigate how the addition of $\mathrm{Ho}_{2} \mathrm{O}_{3}{ }^{4}$ affects the dielectric/physical properties of $\mathrm{HfO}_{2}{ }^{5}$ from not only a high-k engineering point of view but also to learn about the physics of the material. Additionally, high-k epitaxy in which the dielectric material is epitaxial and lattice-matched to the underlying silicon, forming a clean/abrupt interface, is projected as the solution for achieving sub-nanometer electrical functional thickness with minimized leakage currents (standby power), good reliability, and a high electrical breakdown. ${ }^{6}$ Our effort is to stabilize $\mathrm{HfO}_{2}$ in its cubic phase by the addition of $\mathrm{Ho}_{2} \mathrm{O}_{3}$, hoping to achieve functional properties superior than either of the individual end-member precursors. This class of oxide

\footnotetext{
a) Authors to whom correspondence should be addressed. Electronic addresses: shojanpp@gmail.com and rkatiyar@hpcf.upr.edu. Tel.: 787751 4210. Fax: 7877642571.
}

materials can also serve as antireflective/protective coatings and refractory matrices for optical devices, dielectric ceramics for microwave wireless communication devices, ionic conductors, nuclear waste-storage materials, and thermal coatings. ${ }^{7-9}$ In this letter, we present ceramic holmium hafnate as a promising alternative high-k dielectric material, emphasizing its wide bandgap and linear high-k value with low leakage currents.

Polycrystalline powders of HHO were synthesized using high-energy solid state reaction from a stoichiometric mixture (1:1 molar ratio) of $\mathrm{HfO}_{2}$ and $\mathrm{Ho}_{2} \mathrm{O}_{3}$ powders. High purity ( $>99.95 \%$ ) reagents/precursors from Alfa Aesar were pre-fired at $700{ }^{\circ} \mathrm{C}$ in argon atmosphere for about $2 \mathrm{~h}$ to remove water content and other volatile impurities. Mechanical ball milling of stoichiometric amounts of hafnia and holmia was carried out overnight in methanol for fine mixing, followed by calcination in air at $1500^{\circ} \mathrm{C}$ for $24 \mathrm{~h}$, using a Carbolite HTF1700 furnace with heating and cooling rates of $5^{\circ} \mathrm{C} / \mathrm{min}$. The assynthesized powders with $7 \mathrm{wt} . \%$ polyvinyl alcohol were pressed into thick pellets $(\phi=13 \mathrm{~mm}$, thickness $=0.75 \mathrm{~mm})$ at a uniaxial pressure of 4 tons and later sintered at $1550^{\circ} \mathrm{C}$ for 6 h. Phase purity of the sample was checked in slow scan mode $\left(0.25^{\circ} / \mathrm{min}\right)$ with a Rigaku Ultima III X-ray diffractometer (XRD) equipped with $\mathrm{CuK} \alpha$ radiation $(\lambda=1.5405 \AA$ ) source operating in Bragg-Brentano $(\theta-2 \theta)$ geometry at $40 \mathrm{kV}$ and $40 \mathrm{~mA}$. The Rietveld structure refinement of the unit cell and determination of the crystallographic parameters were carried out using a FullProf Suite software package ${ }^{10}$ following Young's strategy. ${ }^{11}$ Local crystal structure analysis was made via temperature-dependent Raman spectroscopy using a Jobin Yvon T64000 spectrometer operating in backscattering configuration and in subtractive mode. About $10 \mathrm{~mW}$ of continuous wave power from a Coherent argon ion laser (Innova 90-5) at $5145 \AA$ was focused to a small spot size of $\sim 2 \mu \mathrm{m}^{2}$. A liquidnitrogen-cooled CCD device collected the Raman scattered signal through an $80 \times$ objective. We collected low and high temperature spectra of the sample in vacuum from $83 \mathrm{~K}$ to 
$1200 \mathrm{~K}$ in steps of $50 \mathrm{~K}$ using Linkam TP93 and TMS94 temperature controllers and liquid nitrogen pump (LNP) cooling module having $\pm 1{ }^{\circ} \mathrm{C}$ accuracy. Room-temperature surface topography of the sample at $3500 \times$ and $10000 \times$ magnifications was analyzed in vacuum using a scanning electron microscope (SEM) having a resolution better than $1 \mu \mathrm{m}$. Elemental analysis of the pellet was carried out by recording the energydispersive X-ray (EDX) spectra. X-ray fluorescence (XRF) spectra were collected to identify and to determine the concentrations of the elements present in the sample. Diffuse reflectance absorption spectra of opaque HHO ceramics were recorded in the spectral window of $190-800 \mathrm{~nm}$ using a Varian Cary UV-Vis spectrophotometer equipped with an integrating sphere. For dielectric and electrical characterization, HHO pellets were DC magnetron sputtered (power density of $\sim 1 \mathrm{~W} /$ $\mathrm{cm}^{2}$ ) at room temperature with $\mathrm{Pt}$ to form the top and bottom electrodes. The resulting $\mathrm{Pt} / \mathrm{HHO} / \mathrm{Pt}$ MIM structures were annealed at $400^{\circ} \mathrm{C}$ in high purity oxygen ambient for proper adhesion of $\mathrm{Pt}$ and recovery of the possible sputter damage. The dielectric and DC leakage current measurements were done under vacuum ( $\sim 10^{-6}$ Torr) using an HP4294A impedance analyzer and Keithley electrometer (model \#6517A). Thermal control was achieved in the range of $82-600 \mathrm{~K}$ using a variable temperature micro-probe system equipped with a programmable temperature controller [MMR Technologies, Inc.]. The samples were kept in the dark during electrical characterization.

The effective cationic radii of 8-fold coordinated $\mathrm{Ho}^{3+}$ in $\mathrm{Ho}_{2} \mathrm{O}_{3}$ and $\mathrm{Hf}^{4+}$ in $\mathrm{HfO}_{2}$ are $1.015 \AA$ and $0.83 \AA^{12}$, respectively, and the corresponding tolerance factor $\left(t_{f}=r_{A^{3+}} / r_{B^{4+}}\right)$ of $1.223(<1.46)^{13}$ predicts the stable formation of $\mathrm{HHO}$ defect (disordered) fluorite structure with higher symmetry (decreased degree of distortion) instead of ordered pyrochlores (increased ion disorder). Figure 1 shows the experimental and Rietveld simulated XRD patterns of HHO powders representing defect fluorite structure belonging to space group $\left(F m \overline{3} m\right.$ or $\left.O_{h}^{5}\right)$ No. 225 , with the sixfoldcoordinated $(\mathrm{Z}=6)$ cations $\left(\mathrm{Ho}^{3+} / \mathrm{Hf}^{4+}\right)$ and the anions $\left(\mathrm{O}^{2-}\right)$ at $4 a(m \overline{3} m$ symmetry) $(0,0,0)$ and $8 c(\overline{4} 3 m$ symmetry $)$ $(0.25,0.25,0.25)$ sites, respectively. The sharp peaks without any superstructure pyrochlore reflections were assigned to their Miller indices with unit cell parameters of the HHO lattice, $a=b=c=5.198 \AA$ and $\alpha=\beta=\gamma=90^{\circ}$. It is worth noting that HHO is found to be a closely lattice-matched oxide with silicon $(5.43 \AA$ ) to enable epitaxial/textured growth on semiconductor. A three-dimensional model of HHO unit cell projected along $c$-axis is illustrated in the inset of Fig. 1, where the $\mathrm{Ho}^{3+} / \mathrm{Hf}^{4+}$ occupies face-centered positions in a cubic unit cell with anions in the eight tetrahedral sites between them. X-ray scattering power of $\mathrm{O}$ is much less than that of rare-earth or transition metal ions $\left(\mathrm{Ho}^{3+} / \mathrm{Hf}^{4+}\right)$, whereas Raman spectroscopy is more sensitive to oxygenmetal vibrations than to metal-metal vibrations and hence is an excellent technique to probe the disorder in the lattice. The factor group analysis for the fluorite structure ${ }^{14}$ with the site symmetry $O_{h}$ for cation and $T_{d}$ for oxygen ion is given by $\Gamma_{O p t}=F_{1 u}^{\text {Infrared }}+F_{2 g}^{\text {Raman }}$ and predicts only one Raman-active mode $F_{2 g}$ originating from the symmetric stretching of oxygen atoms around metal ions in $\mathrm{MO}_{6}$ octahedra. Cubic fluorite phase formation of $\mathrm{HHO}$ was confirmed by room temperature Raman spectroscopy, where a broad phonon mode centered around $300-400 \mathrm{~cm}^{-1}$ spectral region was identified, as shown in Fig. 1(b). The observed large linewidth can be attributed to the static atomic displacement from their ideal positions as a result of lattice strain due to the sample's thermal history and more significantly due to compositional substitution by atoms with mismatched cationic radii and valencies. ${ }^{15}$ The temperature dependent vibrational spectra (data shown for $83 \mathrm{~K}$ and $1200 \mathrm{~K}$ ) depicted in Fig. 1(b) were found to be nearly identical to that obtained at room temperature (with a nominal softening with increasing temperature) confirming that the HHO defect-fluorite phase is quite stable; hence, it is a promising material for various applications in a remarkably wide temperature range. Two more broad bands observed at lower frequencies $\sim 110 \mathrm{~cm}^{-1}$ and $\sim 184 \mathrm{~cm}^{-1}$ may be the normally forbidden zone-boundary acoustic modes appearing due to lattice disorder. With increase in temperature, structural disorder increases and hence the intensity of these peaks is enhanced. HRTEM image of the HHO powder sample given in the inset of Fig. 1(b) reveals the polycrystalline phase formation and illustrates the interplanar $(\mathrm{Ho} / \mathrm{Hf}-$ $\mathrm{Ho} / \mathrm{Hf}$ or O-O) distance of $\sim 2.6 \AA$ along the (X00) plane, in
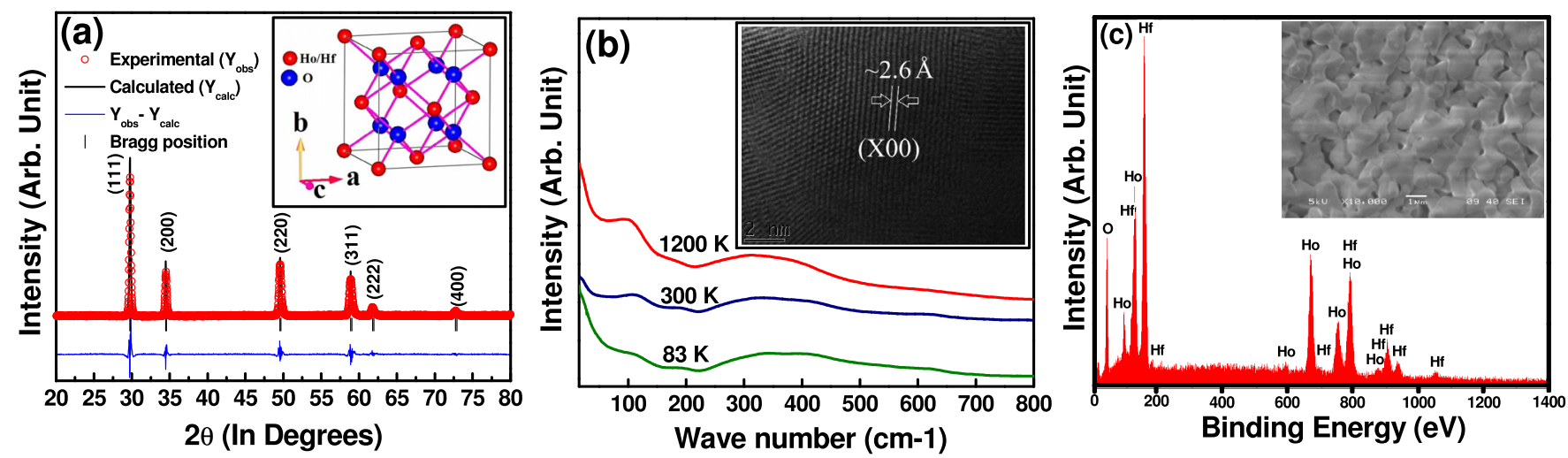

FIG. 1. (a) Rietveld refined room temperature XRD pattern of HHO polycrystalline powders along with Miller indices representing cubic (defect fluorite, $F m \overline{3} m$ or $O_{h}^{5}$ ) symmetry. The inset shows a three-dimensional structure model of HHO unit cell projected along the $c$-axis. (b) Raman spectra of HHO ceramics excited by $514.5 \mathrm{~nm} \mathrm{Ar}+$ laser line at various temperatures. Dark-field high-resolution transmission electron microscope image of HHO powder sample in the inset demonstrates its defect fluorite polycrystalline nature and an inter-ionic (Ho/Hf-Ho/Hf or O-O) distance of $\sim 2.6 \AA$ along the (X00) direction. (c) EDX analysis of HHO pellet and SEM micrograph of the sintered pellet (inset). 


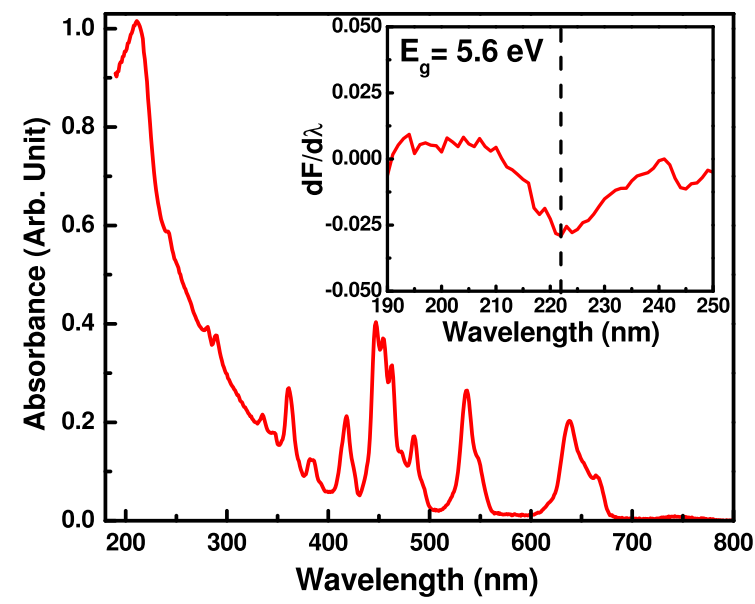

FIG. 2. Absorption spectra of HHO bulk sample from diffuse-reflectance spectroscopy. The vertical line in the first derivative of Kebulka-Munk function with respect to wavelength $(\mathrm{dF} / \mathrm{d} \lambda)$ versus wavelength $(\lambda)$ plot, given in the inset, indicates the inflection point, which lies at $\sim 221 \mathrm{~nm}(\sim 5.6 \mathrm{eV})$.

fairly good agreement with the results obtained from XRD Rietveld analysis. Figure 1(c) is a representative EDX spectrum of the HHO pellet excited by electron beam of low energy of $5 \mathrm{kV}$ (low energy was used to minimize charge accumulation on the insulator sample), showing the presence of all elements (Ho, Hf, and O). On average, the Ho:Hf atomic ratio is nearly $1: 1$ from the intensities of the characteristic lines and it is in good agreement with the composition stoichiometry of holmia and hafnia precursors prior to calcination and with the results obtained from XRF analysis (data not shown). A typical SEM micrograph of the sintered pellet, depicted in the inset of Fig. 1(c), demonstrates fine, dense, and close granular structure with an average grain size of about $2 \mu \mathrm{m}$ and it shows the presence of pores.

Figure 2 shows the diffuse-reflectance absorption spectra of HHO ceramic sample. The absorbance $A$ or the KebulkaMunk function $F$ was deduced from the spectral reflectance data using the Kebulka-Munk relation $A=F=\left(1-R^{2}\right) / 2 R$, where $\mathrm{R}$ is the percentage of reflected light. As shown in the plot $^{16}$ of $(\mathrm{dF} / \mathrm{d} \lambda)$ versus $\lambda$ given in the inset of Fig. 2, diffuse reflectance spectra exhibit an absorption threshold with an inflection point at $\sim 221 \mathrm{~nm}(\sim 5.6 \mathrm{eV})$ that we ascribe to the bandgap. Such a large bandgap will be of interest for electronic device applications if the material has sufficiently enhanced dielectric properties.

Detailed analysis of dielectric and electrical properties of high-k dielectrics is important for their successful application in nanoelectronic devices, such as MOSFET, DRAM, and RRAM. Figure 3(a) and its inset illustrate the frequency dependence of relative dielectric permittivity $\left(\varepsilon^{\prime}\right)$ and loss tangent $(\tan \delta$, where $\delta$ is the phase difference between the applied electric field and the induced current) of polycrystalline HHO high-k dielectrics at zero bias and in the temperature range of 81 to $600 \mathrm{~K}$. The ceramic pellet was cooled to $81 \mathrm{~K}$ and capacitance and loss tangent were measured up to a temperature of $600 \mathrm{~K}$ in steps of $\leq 25 \mathrm{~K}$. The roomtemperature dielectric constant and loss tangent were determined to be $\sim 19-20$ and $\sim 0.001$, respectively. No significant dielectric dispersion (almost linear response) was observed throughout the experimental frequency range. Also, the observed dielectric variation $\left(\Delta \varepsilon^{\prime}\right)$ is small $( \pm 4 \%)$, and the measured loss tangent is less than 0.05 throughout the conventional CMOS device operating temperature of $\sim 200-400 \mathrm{~K}$ and at frequencies that ranged from 1 $\mathrm{kHz}-1 \mathrm{MHz}$. This polar dielectric material has a smooth frequency response (no relaxation) and it is temperature-stable having low-loss. These features are advantageous for gate oxide and DRAM applications. The increase in dielectric loss with temperature is more pronounced at power frequencies $\left(10^{2}\right.$ to $\left.10^{3} \mathrm{~Hz}\right)$ and may be ascribed to thermally generated free carriers which partly contribute to the real part of dielectric permittivity; hence, this parameter slightly increases with temperature. ${ }^{17}$

The absolute complex permittivity is given by the relation $\varepsilon=\varepsilon^{\prime}-j \varepsilon^{\prime \prime}$, where $\varepsilon^{\prime}$ (a measure of how much energy from an external electric field is stored in a material) is the real part and $\varepsilon^{\prime \prime}\left(=\varepsilon^{\prime} \times \tan \delta\right.$, a measure of energy dissipation per period from both dielectric loss and conductivity) is the imaginary part. In Fig. 3(b), we have plotted the temperature response of real and imaginary (inset) parts of relative dielectric permittivity of a polycrystalline HHO pellet in the frequency range of $100 \mathrm{~Hz}-1 \mathrm{MHz} . \varepsilon^{\prime}(\sim 19-20)$ and $\varepsilon^{\prime \prime}(<1)$
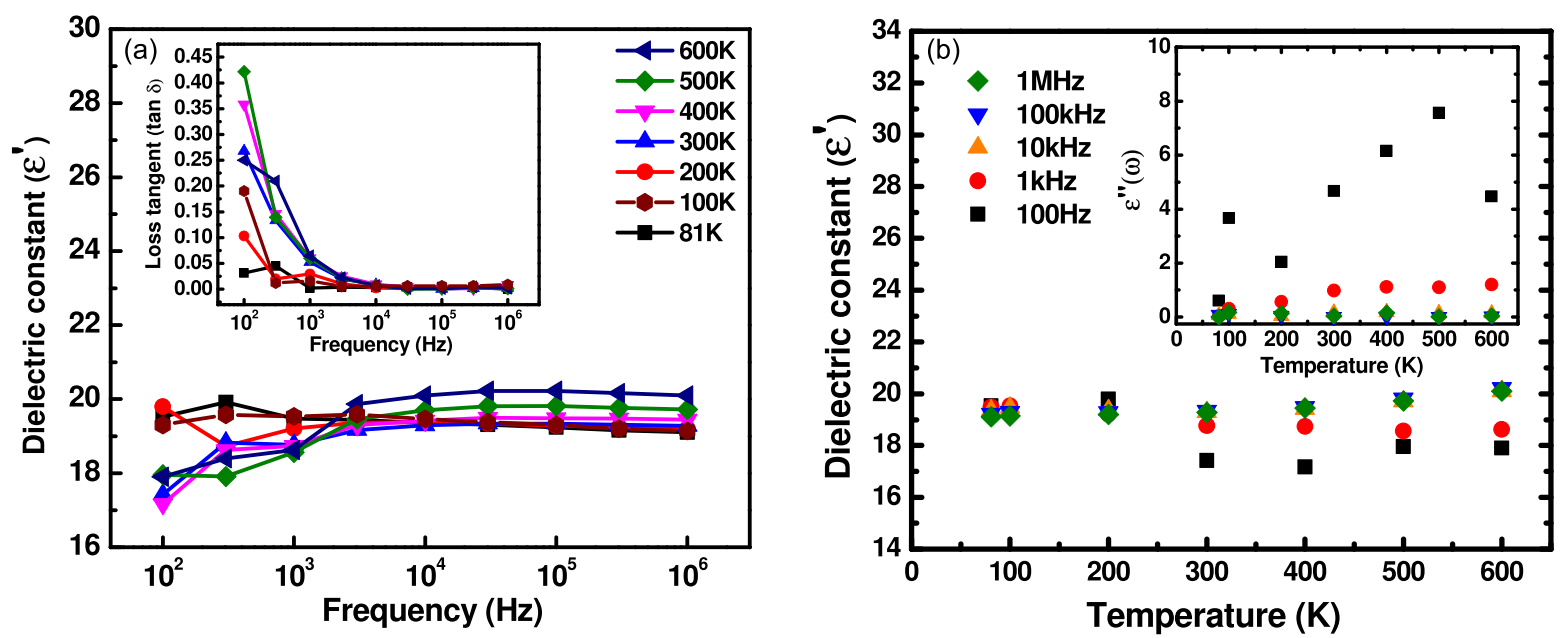

FIG. 3. (a) Frequency dependence of dielectric constant and loss tangent (inset) of polycrystalline HHO dielectrics in the temperature range of $81-600 \mathrm{~K}$. (b) Temperature dependence of real $\left(\varepsilon^{\prime}\right)$ and imaginary $\left(\varepsilon^{\prime \prime}\right)$ (inset) parts of dielectric constant of the sample measured in the frequency range of $100 \mathrm{~Hz}-1 \mathrm{MHz}$. 

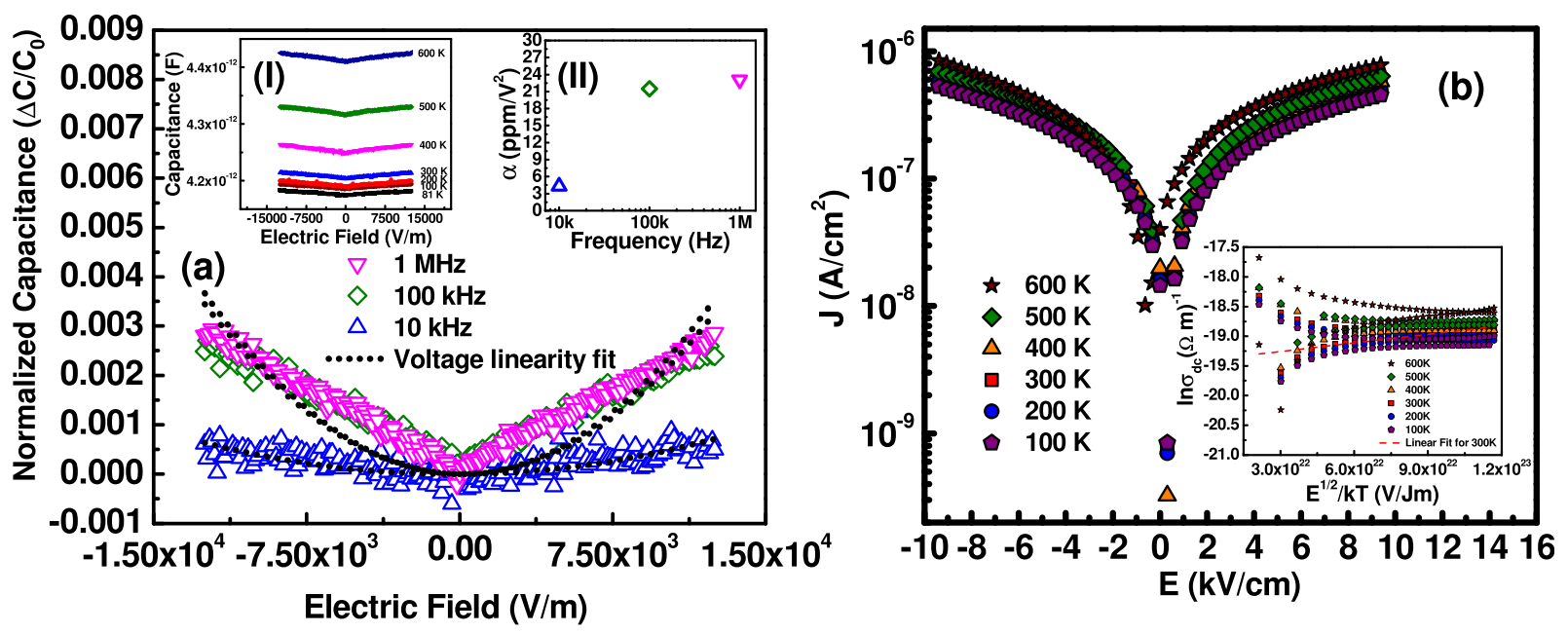

FIG. 4. (a) Applied electric field dependence of normalized capacitance of Pt/HHO/Pt MIM structure at $300 \mathrm{~K}$ for various signal frequencies, along with the respective voltage linearity fits showing good capacitance-voltage linearity of this high-k dielectric. The nominal change in capacitance with temperature measured at $100 \mathrm{kHz}$ is shown in the inset (I). Inset (II) shows the variation in quadratic voltage coefficient as a function of ac- drive signal frequencies. (b) J-E plot for $\mathrm{Pt} / \mathrm{HHO} / \mathrm{Pt}$ capacitor at various temperatures. The inset in Fig. 4 demonstrates the temperature dependence of the dc conductivity at different applied electric fields, along with the Schottky modeling for $300 \mathrm{~K}$ data.

were found to be nearly constant (almost linear without any peak) for experimental frequencies $\geq 1 \mathrm{kHz}$ up to $600 \mathrm{~K}$. This HHO ternary oxide exhibited no dielectric anomaly, that is, in good agreement with the phase stability revealed by Raman spectroscopy studies.

The voltage linearity, a key parameter for high-k materials, can be experimentally verified by modeling the $\mathrm{C}-\mathrm{V}$ plots with the quadratic law ${ }^{18}$

$$
\frac{\Delta C}{C_{0}}=\frac{C_{v}-C_{0}}{C_{0}}=\alpha V^{2}+\beta V,
$$

where $V$ is applied bias voltage; $C_{v}$ and $C_{0}$ are the capacitance at a specific applied voltage and at zero voltage; $\alpha$ and $\beta$ are the quadratic and linear voltage coefficients, expressed in units of parts per million $\left(\mathrm{ppm} / \mathrm{V}^{2}\right)$ and $\mathrm{ppm} / \mathrm{V}$, respectively; and $\Delta C / C_{0}$ is the normalized capacitance. $\alpha$, also known as the voltage coefficient of capacitance (VCC), is a more relevant parameter to be minimized. Electric field dependency of normalized capacitance density of $\mathrm{Pt} / \mathrm{HHO} / \mathrm{Pt}$ MIM structures at $10 \mathrm{kHz}, 100 \mathrm{kHz}$, and $1 \mathrm{MHz}$ evaluated from data obtained from $\mathrm{C}-\mathrm{V}$ measurements is given in Fig. 4(a), together with their voltage linearity fits. A linear dielectric response and little hysteresis were observed while sweeping voltage (electric field) from $-10 \mathrm{~V}(-1.25 \times$ $\left.10^{4} \mathrm{~V} / \mathrm{m}\right)$ to $+10 \mathrm{~V}\left(+1.25 \times 10^{4} \mathrm{~V} / \mathrm{m}\right)$. In other words, $\varepsilon^{\prime}$ is independent of the magnitude of the applied electric field for HHO. There is no dispersion in capacitance with applied bias, confirming the linear dielectric behavior of this electronic material. Inset (II) of Fig. 4(a) shows the variation in positive quadratic VCC $\left(4.3-23 \mathrm{ppm} / \mathrm{V}^{2}\right)$ with ac drive frequency. The temperature dependence $(81-600 \mathrm{~K})$ of capacitance (k-value) measured at $100 \mathrm{kHz}$ showed little change $(-1 \%$ or $+5 \%)$ with respect to the room temperature value, as depicted in inset (I) of Fig. 4(a). The measured quality factor $Q=(1 / \tan \delta)$ was well above the limiting value of 20 at all the measured frequencies (data not shown). This analysis proves that the permittivity of this material has constant value with respect to frequency and temperature for operation over a variety of conditions. These properties reveal the applicability of this material for MIM stacked capacitors, such as radio frequency (RF) coupling and bypass capacitors in oscillators and resonator circuits, filter and analog capacitors in analog/mixed-signal (AMS) circuits, decoupling capacitors for microprocessors (MPUs), and storage capacitors in DRAM and embedded DRAM (eDRAM)/logic devices.

A key motivation in finding an alternative dielectric is to have controlled leakage currents when the material is used either as a gate oxide in logic devices or as a charge storage layer in MIM capacitor structures. Temperature dependent leakage current characteristics were recorded in the dark for $\mathrm{Pt} / \mathrm{HHO} / \mathrm{Pt}$ devices in the $100-600 \mathrm{~K}$ temperature range in order to study their current conduction mechanism(s) and reliability and these are illustrated in Fig. 4(b). The symmetric J$\mathrm{E}$ (current density versus electric field) curves with weak temperature dependence, obtained while ramping the voltage (electric field) from $-750 \mathrm{~V}(-9.38 \mathrm{kV} / \mathrm{cm})$ to $+750 \mathrm{~V}$ $(+9.38 \mathrm{kV} / \mathrm{cm})$, can be ascribed to the similarity in material properties and charge transport mechanisms across the $\mathrm{Pt} /$ $\mathrm{HHO}$ and $\mathrm{HHO} / \mathrm{Pt}$ interfaces. A low leakage current density of $6.12 \times 10^{-7} \mathrm{~A} / \mathrm{cm}^{2}$ was observed at the maximum applied field $(9.38 \mathrm{kV} / \mathrm{cm})$, which validates the charge storage capabilities for its use in future technology nodes. The leakage data were plotted in Schottky coordinates $\left(\ln \sigma_{d c}\right.$ versus $\left.E^{1 / 2} / k T\right)$, as shown in the inset of Fig. 4(b). The expression for Schottky charge transport mechanism ${ }^{19}$ is given by

$$
\sigma=\sigma_{0} \exp \left(\beta E^{1 / 2} / k T\right),
$$

where $\beta=\left(e^{3} / \alpha \pi \varepsilon_{0} \varepsilon_{\infty}\right)^{1 / 2}$, and $\sigma_{0}$ is the low-field conductivity of the system; $E$ is the electric field strength in the insulator; $k$ is the Boltzmann constant; $T$ is absolute temperature; $e$ is electronic charge; $\varepsilon_{0}$ is the permittivity of free space; $\varepsilon_{\infty}$ is electronic permittivity of the insulator; and $\alpha$ is 4 for Schottky emission. A good linear fit in the room temperature Schottky plot [Eq. (2)] is indicative of electrode/interface-limited Schottky conduction mechanism due to field assisted thermionic emission of electrons over a barrier $\Phi_{B}\left(\Phi_{\mathrm{HHO} / \mathrm{Pt}}\right)$, the 
energy offset between Pt Fermi level and HHO conduction band minima. The electronic dielectric constant $\left(\varepsilon_{\infty}\right)$ and high frequency refractive index $\left(n_{\infty}=\sqrt{\varepsilon_{\infty}}\right)$ of HHO estimated from the slope of the linear fit of Schottky plot at $300 \mathrm{~K}$ are found to be 4.29 and 2.07, respectively, and these figures are similar to the values reported for $\mathrm{HfO}_{2},{ }^{20}$ validating our findings. This small value of $\varepsilon_{\infty}$ in comparison with the high dielectric constant (19-20) obtained from dielectric measurements implies that the majority of " $\mathrm{k}$ " must originate from the lattice contribution.

In summary, a defect fluorite structured high-k dielectric $\mathrm{HHO}$ is developed and demonstrated as a potential electronic device material in terms of its structural, optical, charge transport, and temperature- and frequency-independent dielectric properties. A wide bandgap of $5.6 \mathrm{eV}$, a high linear dielectric constant of $\sim 20$, and a low dielectric loss of $\sim 0.001$ were obtained at ambient conditions. The $\mathrm{Pt} / \mathrm{HHO} / \mathrm{Pt}$ capacitor showed very low figures $\left(6.12 \times 10^{-7} \mathrm{~A} / \mathrm{cm}^{2}\right.$ at $9.38 \mathrm{kV} / \mathrm{cm}$ ) for interface-limited Schottky emission leakage currents.

Financial support from DOE Grant No. DE-FG0208ER46526 is acknowledged. S.P.P. is grateful to NSF for financial assistance under Grant No. NSF-EFRI RESTOR \#1038272. Y.S. is thankful to IFN-NSF for doctoral fellowship under NSF-RII-0701525 grant. The authors are thankful to Mr. Oscar Resto and Mr. Salvador Gavalda for their help in conducting HRTEM experiments and diffuse reflectance spectroscopy measurements, respectively.
${ }^{1}$ G. E. Moore, Electron. Mag. 38, 8 (1965).

${ }^{2}$ International Technology Roadmap for Semiconductors (Semiconductor Industry Association, San Jose, CA, 2010), see http:/www.itrs.net for updates.

${ }^{3}$ G. D. Wilk, R. M. Wallace, and J. M. Anthony, J. Appl. Phys. 89, 5243 (2001).

${ }^{4}$ R. D. Shannon, J. Appl. Phys. 73, 348 (1993).

${ }^{5}$ B. H. Lee, L. Kang, R. Nieh, W.-J. Qi, and J. C. Lee, Appl. Phys. Lett. 76, 1926 (2000).

${ }^{6}$ A. Laha, H. J. Osten, and A. Fissel, Appl. Phys. Lett. 90, 113508 (2007).

${ }^{7}$ R. Chow, S. Falabella, G. E. Loomis, F. Rainer, C. J. Stolz, and M. R. Kozlowski, Appl. Opt. 32(28), 5567 (1993).

${ }^{8} \mathrm{~N}$. McN. Alford, in Dielectric Materials for Wireless Communication, edited by M. T. Sebastian (Elsevier, Amsterdam, 2008), p. xi.

${ }^{9}$ R. C. Ewing, W. J. Weber, and J. Lian, J. Appl. Phys. 95, 5949 (2004).

${ }^{10}$ J. Rodríguez-Carvajal, Physica B 192, 55 (1993) and J. RodriguezCarvajal Laboratory, FULLPROF 2012, Laboratoire Leon Brillouin, CEACNRS, France, see http://www.ill.eu/sites/fullprof/.

${ }^{11}$ S. P. Pavunny, A. Kumar, P. Misra, J. F. Scott, and R. S. Katiyar, Phys. Status Solidi B 251, 131 (2014).

${ }^{12}$ R. D. Shannon, Acta Crystallogr., Sect. A: Cryst. Phys., Diffr., Theor. Gen. Crystallogr. 32, 751 (1976).

${ }^{13}$ M. A. Subramanian, G. Aravamudan, and G. V. Subba Rao, Prog. Solid State Chem. 15, 55 (1983).

${ }^{14}$ N. Kjerulf-Jensen, R. W. Berg, and F. W. Poulsen, in Proceedings of the Second European Solid Oxide Fuel Cell Forum, edited by B. Thorstensen (European Solid Oxide Fuel Cell Forum, Norway, 1996), Vol. 2, p. 647.

${ }^{15}$ N. J. Hess, B. D. Begg, S. D. Conradson, D. E. McCready, P. L. Gassman, and W. J. Weber, J. Phys. Chem. B 106, 4663 (2002).

${ }^{16}$ B. Gilbert, C. Frandsen, E. R. Maxey, and D. M. Sherman, Phys. Rev. B 79, 035108 (2009).

${ }^{17}$ S. P. Pavunny, R. Thomas, A. Kumar, N. M. Murari, and R. S. Katiyar, J. Appl. Phys. 111, 102811 (2012).

${ }^{18}$ S. Blonkowski, Appl. Phys. Lett. 91, 172903 (2007).

${ }^{19}$ J. G. Simmons, Phys. Rev. 155, 657 (1967).

${ }^{20}$ A. A. Demkov and A. Navrotsky, Materials Fundamentals of Gate Dielectrics (Springer, New York, 2005). 